\title{
The Research on Sports Training Aids System Based on Data Warehouse Technology
}

\author{
LIU Yan ${ }^{1,}$ a, LIU Xiaofang ${ }^{2}$ \\ 1,2 Jiangxi college of foreign study,NanChang,330099, China \\ aliuyan126@yeah.net
}

\section{Keywords: Sports Training; Aids System; Data Warehouse}

\begin{abstract}
With the rapid development of computer technology and the business community have made new demands, data warehouse technology is introduced. Traditional database technology is a single source of data, that database will be mainly engaged in transaction processing, batch processing to decision analysis and other types of data processing work, especially the development of decision support systems, decision support systems in sports training field has been applied, with the emergence of data warehouse and data mining technology and development, but also these two technologies applied to them. This article describes the use of data warehouse and data mining technology to build college sports training aid decision support system to realize the different aspects of the organic integration of college students, the training of scientific theory, advanced training methods applied to university sports training management, combined User input to generate a reasonable sports training programs. The system is based Nanchang University undergraduates of sports scores and physical examination tables and other data, build a data warehouse cubes, and data mining techniques applied to them.
\end{abstract}

\section{Introduction}

In recent years, because of data compression, mass storage, broadband networks, high-speed computer technology, making multimedia technology will soon become a hot computer and communications industries, and one of the important directions databases as an effective means of information management has become a multimedia research. Computer multimedia technology is high-tech in recent years, which allows information to text, images, sound and animation and other forms of performance, and the ability to save these multimedia information, management, processing and transmission [1-2]. Multimedia database multimedia information stored in the computer database, with a unified mechanism to manage and control data to ensure safe and correct data, and to the use and maintenance of data has brought great convenience.

In the sports field, this is even more important to build a multimedia database. Because of the characteristics of competitive sports, coaches need to analyze large amounts of video information, draw the information they need to guide the athletes training [3]. This requires that the video data can be unified management, data acquisition becomes concise and easy. Sports action is visual performance sports, is the foundation of all sports. In the computer, in order to clearly describe the features and processes sports action, we need to use text, motion pictures and animation, and even have a soundtrack commentary, which requires the multimedia database and multimedia information processing technology. In sports, coaches often need a variety of technical movements opposing player statistical analysis draw many instructional knowledge to guide our athletes' training and competition. This statistical data is often great, just do a simple analysis is difficult to obtain knowledge of instructional needs. .

\section{The related theory and technology analysis}

As a high-end data warehousing database extension technology has become the hot spot of society. A data warehouse is a subject-oriented, integrated, stable, time-varying data sets to support management decision-making processes. Data warehouse data source data (including operating and external market data, work data, file data, database, etc.) is collected, and standardization, filtration, 
purification, covering timestamp data warehouse (data warehouse), then Through a variety of tools (OLAP tools, reporting tools, DSS tools, data mining tools, etc.) for data warehouse data knowledge discovery for customers and applied in practice, provide theoretical support for scientific decision-making. Data warehouse system contains a complete decision support system for the purpose of data warehousing, OLAP and data mining entity that can implement specific for specific industries and specific companies [4--5]. Figure 1 is a general block diagram of a data warehousing system.

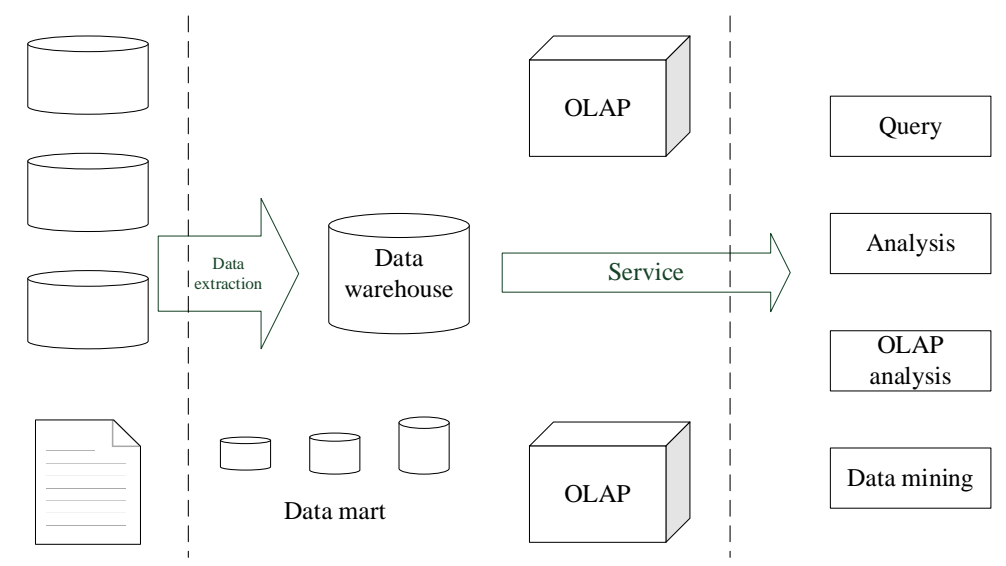

Figure 1. System structure of the data warehouse

Data Organization Data Warehouse consists of three, namely virtual storage, mining method based on multi-dimensional database stored in the memory storage of relational tables and multidimensional database directly facing the organization in the form of data, data analysis required for the operation, it DW angle large amounts of data from client slicing side interest, abstract, and set the appropriate spatial indexing and data warehouse metadata management file corresponding to the data and virtual storage, tissue relationship is more complex compared to relational tables based storage method is more suitable for the organization, in a data warehouse storing large amounts of data [6-7].

Data warehouse is a subject-oriented, integrated, time-varying, to better support decision analysis data collection, the main features are as follows: (1) data warehouse is a subject-oriented. Traditional database-oriented application organized, low degree of correlation between the data, consider how the design is more beneficial to operate the data (add, delete, change, query), its purpose is to handle transaction processing, improving daily speed and accuracy of transaction processing. The main purpose of a data warehouse is a complex transaction analysis, which depart from the overall transaction, subject-oriented organizational data. Topic is a higher level of data classification standards, each topic corresponds to a macro analysis field analysis objects involved. Subject-oriented organization can maximize data, access to the required information. (2) The data warehouse is integrated. Data warehouses can integrate data from various sources. Data from different sources before entering the data warehouse must be processed, unified data structure and coding, unified raw data inconsistencies, like the name of different meaning, different name synonymous etc., and the structure for application to convert from subject-oriented. (3) The data warehouse is not updatable. The main data warehouse for decision analysis, data manipulation involved mainly data query. It reflects the content within a fairly long period of historical data, the most recent data, rather than routine transaction processing generated data warehouse system periodically extracted from the data source in the new data add to the data warehouse into a data warehouse after little or no Update without modification. (4) The data warehouse is changing with time. Since the data warehouse to provide analytical support, the need to ensure the current data is the latest and most valuable, it contains the key code data entry time, indicating the historical period of the data. This is suitable for time trend analysis. 


\section{Data warehouse design for sport training}

System by function can be divided into multimedia database and technology statistics and analysis of two modules. Structure shown in Figure 2.

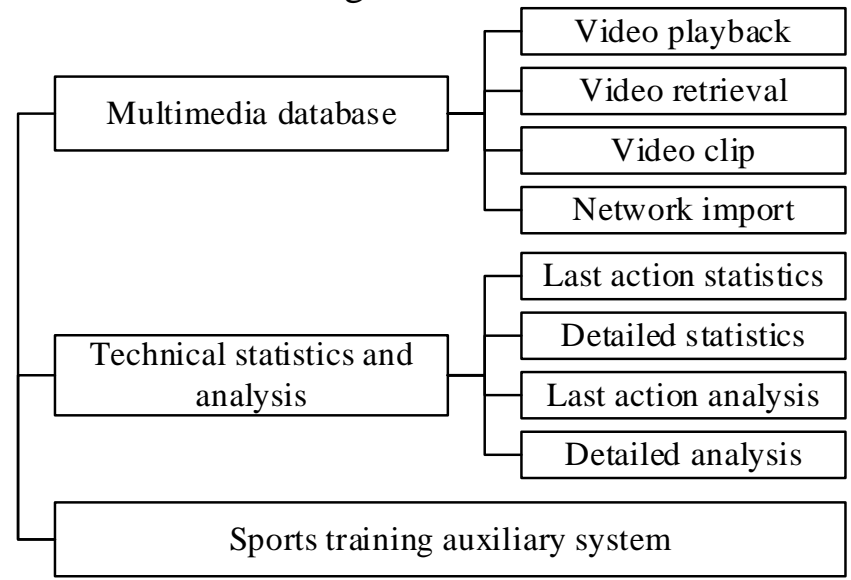

Figure 2. System structure of the data warehouse

Users can select a particular athlete or class of video data according to their needs the game to be played. When playing the user can freely choose certain playback speed. The system also provides users pause and single frame playback function. Technical Data Editor enables users to use the tools provided to certain technical movements of a player a technical stage of the clip down into the database. The score data editing can be divided into route information to short segment will become a divided unit data.

The data warehouse design uses the popular star schema. Star model is based on relational data warehouse, it is facing a multidimensional online analytical data organization. Relational structure can better adapt to the multi-dimensional data representation and storage. In the case of university students' basic body as the fact table, and select the associated four dimensions, each dimension table has its own attributes, dimension by dimension and fact tables associated with the keyword. Its star model in Figure 2.

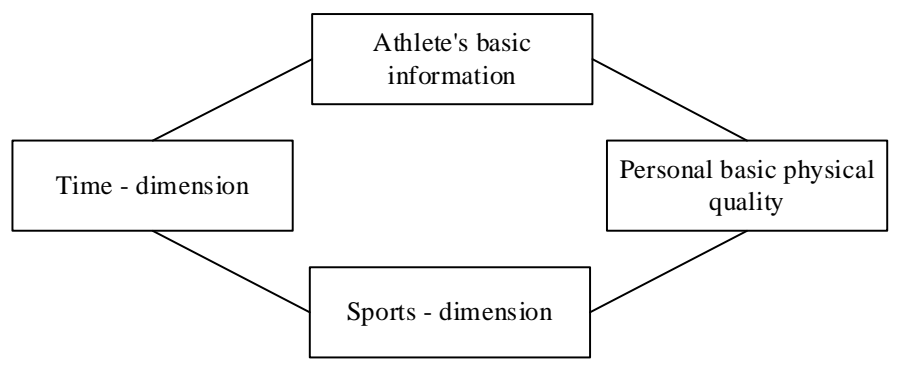

Figure 3. Star model

Data warehouse management system for decision support rather than transaction processing and design, so that each time the user wants to perform data mining, decision support will only extract useful data out from the operating environment and input to the data warehouse. After the input is complete, the data warehouse becomes read-only, only under special conditions can be modified.

\section{Design and implementation of data storage structure of sports training}

In the star type model, metrics and code values for each dimension of facts table is located in the center of the star, the description of each dimension composing dimension tables located star angle, dimensional code (including time code, project code, athletes numbers, coaches No, the game level code, grade category code) as a public code keys for each dimension tables linked. If the information contained too complex dimension, you need to be analyzed by levels or classes, or to learn the details of the data, it will extend outwardly these dimensions and levels or categories of information, details linked. Star sports performance analysis model is shown in Figure 4. 


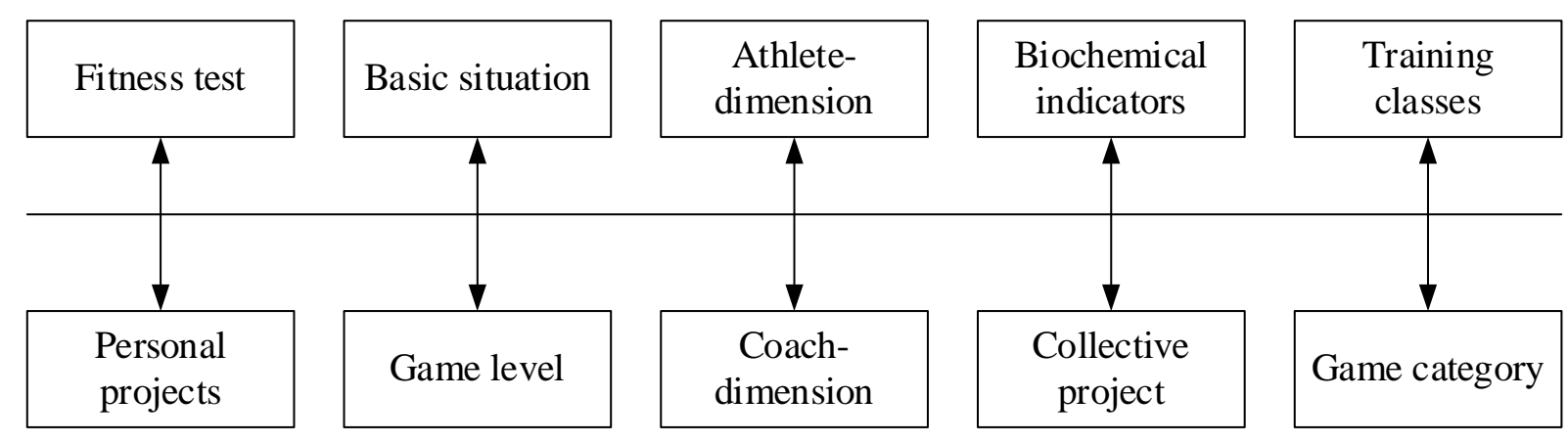

Figure 4. Relationship of sports training aids analysis system Based on Data Warehouse

In the data warehouse database management system is achieved by a series of SQL Server 2000 data table. For each topic from the star center to expand the definition of the model, the establishment of a fact table (including all metrics and code values for each dimension), each dimension build a dimension table (dimension table that contains detailed information about the code level dimension or category) and the appropriate level or class table (including all levels or categories of code), Detailed tables (contains detailed information). Each record in the fact table contains a pointer pointing to each dimension (as a dimension table's primary key), table level or category in the table point to each record containing the details of each table pointer (as the main details of the table keywords). These pointers will associate fact table and dimension table, level or category table, detail tables together, merging in a theme, it is easy to implement multidimensional query and statistical data, and thus facilitate the analysis of personnel from different angles and at different levels of training case study, When such analysis is only related to the main information dimension, you can simply query the dimension tables, thereby increasing the processing speed; if you want to understand the details, it searches through the dimension table in the details table.

\section{Conclusion}

Physical education is an important part of school education, how to make computer and network technology services in daily physical education in schools is a necessary requirement in today's information age. Science and training is an urgent need for the development trend in today's competitive sports, the sport constitute a prerequisite for a breakthrough performance. Sports, especially athletic confrontational, generally requires not only a high technical level of athletes, while tactics are also very important. In college sports training data analysis process, using data warehouse and data mining technology, on the one hand according to the athlete's physical fitness test results on a regular basis, and the different testing standards, the conversion of each athlete's physical fitness test results, identify gaps Players and so develop appropriate coaching program. On the other hand, similar to soccer, volleyball, basketball and other confrontational campaign, data mining technology based on records every game events (score, offense, turnovers, fouls, etc.) statistics, analysis of the opponent's weaknesses, so as to formulate the more effective tactics.

\section{Reference}

[1] Chen Y. Study on the Application of Data Mining-Based BP Neural Network Forecasting Model in Physical Training[J]. International Journal of Digital Content Technology \& its Applications, 2012, 6(17).

[2] Chen Y J, Hung Y C. Using real-time acceleration data for exercise movement training with a decision tree approach[J]. Expert Systems with Applications, 2010, 37(12): 7552-7556.

[3] Kantardzic M. Data mining: concepts, models, methods, and algorithms[M]. John Wiley \& Sons, 2011.

[4] Conroy K, May G, Roantree M, et al. Knowledge acquisition from sensor data in an equine environment[M]//Data Warehousing and Knowledge Discovery. Springer Berlin Heidelberg, 2011: 
432-444.

[5] Greenes R A, Shortliffe E H. Commentary: Informatics in biomedicine and health care[J]. Academic Medicine, 2009, 84(7): 818-820.

[6] Wang Q C, Wu H J. Design Research in Motion cloud computing platform based on a training aid system[C]//Applied Mechanics and Materials. 2014, 635: 1551-1554.

[7] Tan Y, Kang T. Research on Detection of Moving Target Based on Hybrid Algorithm Min-ing Method for Sports Video Analysis[J]. Open Cybernetics \& Systemics Journal, 2015, 9: 2456-2462. 